\title{
Collective motion and oscillator synchronization
}

\author{
Rodolphe Sepulchre ${ }^{\ddagger}$, Derek Paley ${ }^{2}$, and Naomi Leonard ${ }^{\S 2}$ \\ 1 Department of Electrical Engineering and Computer Science, Université de \\ Liège, Institut Montefiore B28, B-4000 Liège, Belgium. r.sepulchre@ulg.ac.be \\ 2 Department of Mechanical and Aerospace Engineering, Princeton University, \\ Princeton, NJ 08544 USA. \{naomi $\},\{$ dpaley\}@princeton.edu
}

Summary. This paper studies connections between phase models of coupled oscillators and kinematic models of groups of self-propelled particles. These connections are exploited in the analysis and design of feedback control laws for the individuals that stabilize collective motions for the group.

\section{Introduction}

Synchronization of a large population of oscillators at a common pulsation is a popular phenomenon in physics (e.g. in large arrays of superconducting Josephson junctions) and in biology (e.g. in large networks of coupled neurons). Likewise, collective motion is commonly observed in large populations of natural organisms such as flocks of birds or schools of fish. These two instances of "self-organization" in dynamical systems have been widely studied in the literature. The convergence to organized formations for the group starting from arbitrarily disorganized initial conditions for the individuals is robust to small differences between the individuals and is attributed to the weak interactions (coupling) between the individual dynamics. For instance, synaptic connections between neurons influence their individual firing rate; likewise, a fish adapts its direction of motion to avoid collisions with neighbors.

Mathematically, oscillator synchronization and collective motion have been studied - quite independently from one another - through simplified dynam-

\footnotetext{
$\ddagger$ This paper presents research partially supported by the Belgian Programme on Inter-university Poles of Attraction, initiated by the Belgian State, Prime Minister's Office for Science, Technology and Culture. The research was partially completed during the sabbatical stay of the author at Princeton University.

$\S$ Research supported in part by the Office of Naval Research under grants N0001402-1-0826 and N00014-02-1-0861, by the National Science Foundation under grant CCR-9980058 and by the Air Force Office of Scientific Research under grant F49620-01-1-0382.
} 
ical models that assume (nearly) identical and low-dimensional dynamics for the uncoupled systems but nevertheless exhibit rich and complex dynamics under weak coupling. The goal of the present paper is to exploit the analogy between these simplified models. More specifically, we develop analysis and design tools for the stabilization of collective motions based on available results for coupled oscillators. The development of systematic analysis design tools for collective motion is motivated by the expanding number of engineering applications that require coordination of many "individuals" to achieve a prescribed mission. The results of the present paper are specifically motivated by coordinated motions in groups of underwater vehicles viewed as reconfigurable moving sensor arrays for data collection in the ocean [II03].

The particle model we consider in this paper is a (kinematic) model of self-propelled particles recently introduced in [JK02]. Each particle moves at constant speed in the plane but adapts its orientation (i.e. the direction of its unit velocity vector) according to the motion of neighboring particles. The state space of each particle is the group $S E(2) \approx \mathbb{R}^{2} \times S^{1}$ of rigid displacements in the plane. The system of $N$ coupled particles thus evolves on a high-dimensional state space ( $N$ copies of $S E(2))$. However, when the coupling only includes relative orientation variables and disregards relative spacing variables, the spatial variables can be ignored and the reduced dynamics evolve on $N$ copies of $S^{1}$, i.e. an $N$-dimensional torus. The reduced dynamics then become equivalent to phase models of $N$ coupled oscillators in which each oscillator is only modelled by a phase variable.

Remarkable analysis results have been obtained by Watanabe and Strogatz [WS94] for phase models of coupled oscillators under the particular assumption of (all-to-all) pure sinusoidal coupling. They showed that this model possesses $N-3$ constants of motion and provided a global analysis of the remaining low-dimensional dynamics. The oscillators either asymptotically synchronize or converge to a manifold of "incoherent" states. These incoherent states are characterized by an arbitrary distribution of phase differences on the unit circle such that the centroid of the oscillators vanishes. In our particle model, phase synchronization corresponds to parallel motion of the moving particles, whereas a vanishing centroid of the oscillators corresponds to motion of the particles around a fixed center of mass. Sinusoidal coupling of the orientation (phase) variables thus stabilizes the linear momentum of the group; either to a maximal value (parallel motion) or to a minimal value (fixed center of mass).

Additional control is needed to stabilize the spacing variables. To retain the results of the phase model of Watanabe and Strogatz, we enforce a time-scale separation between the orientation dynamics and the spacing dynamics. The phase oscillator model captures the fast dynamics and guarantees convergence of the solutions to an invariant slow manifold. For parallel motion, the slow manifold has codimension $N-1$. Small deviations from the parallel motion are used to control the relative spacing of the particles to prescribed formations. For motions around a fixed center of mass, the slow manifold has codimension 
2: the design on the slow manifold is the original design constrained to a fixed position for the center of mass. This constraint simplifies the design of control laws that stabilize formations around a fixed center of mass, such as circular motion of all the particles on a fixed circle.

The connection between the phase-oscillator model of Watanabe and Strogatz [WS94] and the particle model of Justh and Krishnaprasad [JK02] is thus exploited to decouple the design of relative orientations from the design of relative spacing in particle models. The resulting dynamics have provable global convergence properties, and the proposed methodology should be applicable beyond the simplest stabilization problems considered in this paper. The last section of the paper summarizes limitations of the present approach and possible directions for future research.

\section{Models of self-propelled particles}

We consider a continuous-time kinematic model of $N$ identical particles (of unit mass) moving in the plane at unit speed [JK02]:

$$
\begin{aligned}
& \dot{r}_{k}=e^{i \theta_{k}} \\
& \dot{\theta}_{k}=u_{k}, \quad 1 \leq k \leq N .
\end{aligned}
$$

In complex notation, the vector $r_{k} \in \mathbb{C} \approx \mathbb{R}^{2}$ denotes the position of particle $k$ and the angle $\theta_{k} \in S^{1}$ denotes the orientation of its (unit) velocity vector $e^{i \theta_{k}}=\cos \theta_{k}+i \sin \theta_{k}$. We omit the index of a coordinate to denote the corresponding $N$-vector, e.g. $r=\left(r_{1}, \ldots, r_{N}\right)^{T}$. In the absence of steering control $\left(\dot{\theta}_{k}=0\right)$, each particle moves at unit speed in a fixed direction and its motion is decoupled from the other particles. We study the influence of various feedback control laws that result in coupled dynamics and closed-loop convergence to different types of organized or collective motion. We assume identical control for each particle. In that sense, the collective motions that we analyze in the present paper do not require differentiated control action for the different particles (e.g. the presence of a leader for the group). For convenience, we decompose the steering control of particle $k$ into the sum of two terms:

$$
u_{k}=u_{k}^{s p a c}+K u_{k}^{a l i g n} .
$$

The term $u_{k}^{\text {align }}$ depends only on relative orientation, i.e., on the variables $\theta_{i j}=\theta_{i}-\theta_{j}, 1 \leq i, j \leq N$. The term $u_{k}^{s p a c}$ depends both on relative orientation and relative spacing, i.e., on the variables $\theta_{i j}$ and $r_{i j}=r_{i}-r_{j}, 1 \leq i, j \leq N$. The simplest form of phase coupling,

$$
u_{k}^{\text {align }}=\frac{1}{N} \sum_{j=1}^{N} \sin \left(\theta_{j}-\theta_{k}\right),
$$


is adopted throughout the paper, while different choices are discussed for the feedback $u_{k}^{s p a c}$. The sign of the parameter $K$ plays an important role in the results of the paper.

The kinematic model (1) has been recently studied by Justh and Krishnaprasad [JK02, JK03]. These authors have emphasized the Lie group structure that underlies the state space. The configuration space consists of $N$ copies of the group $S E(2)$. When the control law only depends on relative orientations and relative spacing, it is invariant under an action of the symmetry group $S E(2)$ and the closed-loop dynamics evolve on a reduced shape space. Equilibria of the reduced dynamics correspond to equilibrium shapes and can be only of two types [JK02]: parallel motions, characterized by a common orientation for all the particles (with arbitrary relative spacing), and circular motions, characterized by circular orbits of the particles around a fixed point. Both types of motion have been observed in simulations in a number of models that are kinematic or dynamic variants of the model (1), see for instance [LRC01].

A simplification of the model (1) occurs when the feedback laws depend on relative orientations only $\left(u_{k}^{s p a c} \equiv 0\right)$. The control has then a much larger symmetry group ( $N$ copies of the translation group), and the reduced model becomes equivalent to phase models of the form

$$
\dot{\theta}_{k}=\omega_{k}+\sum_{j} u_{j k}\left(\theta_{j}-\theta_{k}\right), \quad k=1, \ldots, N
$$

where the phase variable $\left(\theta_{1}, \ldots, \theta_{N}\right)$ belongs to the $N$-dimensional torus $T^{N}$. (The model (4) still has an $S^{1}$-symmetry because the feedback only depends on phase differences). Formal equivalence with (1)-(2) is obtained with $u_{k}^{s p a c}=\omega_{k}$ and $K u_{k}^{\text {align }}=\sum_{j} u_{j k}\left(\theta_{j}-\theta_{k}\right)$. In the absence of coupling $\left(u_{i j} \equiv 0\right.$ for all $i$ and $j$ ), each particle "rotates" at its natural frequency $\omega_{i}$ around a fixed point. The phase coupling function $u_{i j}$ between oscillator $i$ and oscillator $j$ is assumed to be continuously differentiable and $2 \pi$-periodic. The choice (3) assumes all-to-all coupling with an identical phase-difference coupling function that only includes one harmonic.

Phase-oscillator models of the type (4) have been widely studied in the neuroscience and physics literature. They represent a simplification of more complex models (for instance of neurons) in which the uncoupled oscillator dynamics each have an attracting limit cycle in a higher-dimensional state space. Under the assumption of weak coupling, the reduction of higher-dimensional models to (4) by asymptotic methods (singular perturbations and averaging) has been studied in e.g. Ermentrout and Kopell [EK90] and Hoppensteadt and Izhikevich [HI97].

Even with the simplest type of coupling (3), the phase model (4), then referred to as the Kuramoto model [Kur84], exhibits rich dynamics and has received considerable attention in the literature. Analytical results have been obtained for the continuous limit of $N \rightarrow \infty$ (see Strogatz [Str00] for a recent 
review). In the finite case, results are mostly restricted to the situation of identical natural frequencies: $\omega_{k}=\omega, 1 \leq k \leq N$. (The common frequency $\omega$ can be set to zero without loss of generality by rewriting (4) in a rotating frame). The main results for the latter case have been obtained by Watanabe and Strogatz in [WS94]). These are and reviewed in the next section for their relevance to the present framework.

Our goal is to make the available results for phase models relevant to the particle model (1), even when the control $u_{k}^{\text {spac }}$ is no longer constant. The proposed approach is to assume a large enough value for the parameter $K$ such as to enforce a time-scale decomposition between the (fast) orientation dynamics (determined by the phase model (4)) and the (slow) spacing dynamics determined by the particle model (1) restricted to its slow manifold. Thus, we study the singularly perturbed model

$$
\begin{aligned}
\dot{r}_{k} & =e^{i \theta_{k}} \\
\epsilon \dot{\theta}_{k} & =\epsilon u_{k}^{s p a c}+\frac{1}{N} \sum_{i=1}^{N} \sin \left(\theta_{i}-\theta_{j}\right), \quad 1 \leq k \leq N,
\end{aligned}
$$

where the small parameter $\epsilon$ enforces a time-scale separation between fast dynamics in the time-scale $\tau=\frac{t-t_{0}}{\epsilon}$ and slow dynamics in the time-scale $t$. In the fast time-scale $\tau$, the variable $r$ is frozen and the dynamics reduce to

$$
\frac{d}{d \tau} \theta_{k}=\epsilon u_{k}^{s p a c}+\frac{1}{N} \sum_{i=1}^{N} \sin \left(\theta_{i}-\theta_{j}\right)
$$

which, in the limit $\epsilon=0$, is precisely the phase model

$$
\dot{\theta}_{k}=\frac{1}{N} \sum_{i=1}^{N} \sin \left(\theta_{i}-\theta_{j}\right), \quad 1 \leq k \leq N
$$

studied by Strogatz and Watanabe. In the decomposition (2) of the feedback control, the control term $u_{k}^{\text {align }}$ will determine the fast dynamics whereas the control term $u_{k}^{s p a c}$ will affect the slow dynamics only.

Euler discretization of the continuous-time model (1) yields the discretetime equations

$$
\begin{aligned}
& r_{k}(t+1)-r_{k}(t)=e^{i \theta_{k}} \\
& \theta_{k}(t+1)-\theta_{k}(t)=u_{k} \quad 1 \leq k \leq N .
\end{aligned}
$$

The direction of motion of particle $k$ is updated at each time step according to some feedback control $u_{k}$. We consider continuous-time models in this paper, but we mention a few relevant references to study their discrete-time counterpart. Couzin et al. $\left[\mathrm{CKJ}^{+} 02\right]$ have studied such a model where the feedback control is determined from a set of simple rules: repulsion from close neighbors, attraction to distant neighbors, and preference for a common orientation. Their model includes stochastic effects but also exhibits collective motions reminiscent of either parallel motion or circular motion around a fixed center of mass. Interestingly, these authors have shown coexistence of these 
two types of motion in certain parameter ranges and hysteretic transition from one to the other. A discrete version of (4) has been studied by Viczek et al. $\left[\mathrm{VCBJ}^{+} 95\right]$, in which $\omega_{k}$ is a random variable.

\section{Watanabe-Strogatz analysis of phase-oscillator networks}

This section summarizes the analysis of Watanabe and Strogatz [WS94] for the phase-oscillator model

$$
\dot{\theta}_{k}=\omega_{k}+\frac{K}{N} \sum_{j=1}^{N} \cos \left(\theta_{j}-\theta_{k}-\delta\right), k=1, \ldots, N, \quad 0<\delta<\pi .
$$

They obtained the model (8) with $\omega_{k} \equiv 0$ by averaging a model of coupled Josephson junctions. The parameter $\delta$ plays no role in the present paper and is set to $\pi / 2$ in the control law (3). The results summarized below are instrumental in our analysis of the particle model (1).

We start by introducing the notation

$$
p_{\theta}=\frac{1}{N} \sum_{k=1}^{N} e^{i \theta_{k}}\left(=\frac{1}{N} \sum_{k} \dot{r}_{k}\right)
$$

for the centroid of oscillators in the model (8), or, equivalently, the linear momentum of the group of particles in the model (1). The codimension-two manifold defined by the algebraic condition $p_{\theta}=0$ plays an important role throughout the paper and will be termed the balanced manifold (individual motions of particles balance in this manifold to result in a fixed position for the center of mass of the group). ${ }^{3}$

In complex notation, we set

$$
p_{\theta}=g+i h=\left|p_{\theta}\right| e^{i \phi}
$$

with $g=\frac{1}{N} \sum_{k=1}^{N} \cos \theta_{k}$ and $h=\frac{1}{N} \sum_{k=1}^{N} \sin \theta_{k}$ and rewrite (8) as

$$
\dot{\theta}_{k}=\omega_{k}+K\left(g \cos \left(\theta_{k}+\delta\right)+h \sin \left(\theta_{k}+\delta\right)\right), k=1, \ldots, N
$$

or

$$
\dot{\theta}_{k}=\omega_{k}+K\left|p_{\theta}\right| \operatorname{Re}\left(e^{i\left(\phi-\theta_{k}-\delta\right)}\right), k=1, \ldots, N
$$

\footnotetext{
${ }^{3}$ In the literature of phase oscillators, it has been termed the incoherent state manifold because $N-2$ phase differences are arbitrary in this manifold in contrast to the synchronized state in which all phase differences vanish.
} 


\subsection{Change of coordinates}

The key of the analysis in [WS94] is the change of coordinates

$$
\tan \frac{\theta_{k}-\Theta}{2}=\sqrt{\frac{1+\gamma}{1-\gamma}} \tan \frac{\psi_{k}-\Psi}{2}, k=1, \ldots, N
$$

which maps the $N$-dimensional state vector $\left(\theta_{1}, \ldots, \theta_{N}\right)$ to the $(N+3)$ dimensional state vector $\left(\gamma, \Theta, \Psi, \psi_{1}, \ldots, \psi_{N}\right)$. Equation (12) should be regarded as a way to redistribute the phase variables $\theta_{k}$ on the unit circle. The new phase variables $\psi_{k}$ result from a time-varying but uniform transformation that involves two rigid rotations (of angle $\Theta$ and $\Psi$ respectively) and one dilation (measured by the variable $0 \leq \gamma<1$ ). Differentiating (12) with respect to time and using (10) results in the identities

$$
\begin{aligned}
& 0=\sqrt{1-\gamma^{2}} \dot{\psi}_{k}-\left(1-\gamma \cos \left(\psi_{k}-\Psi\right)\right) \omega_{k}+ \\
& +\left[\dot{\Theta}-\sqrt{1-\gamma^{2}} \dot{\Psi}+K(g \gamma \cos (\Theta+\delta)+h \gamma \sin (\Theta+\delta))\right] \\
& +\cos \left(\psi_{k}-\Psi\right)[-\gamma \dot{\Theta}-K(g \cos (\Theta+\delta)+h \sin (\Theta+\delta))] \\
& +\sin \left(\psi_{k}-\Psi\right)\left[\frac{\dot{\gamma}}{\sqrt{1-\gamma^{2}}}+K \sqrt{1-\gamma^{2}}(g \sin (\Theta+\delta)-h \cos (\Theta+\delta))\right], \quad 1 \leq k \leq N .
\end{aligned}
$$

It is then easily observed that if the three variables $(\gamma, \Theta, \Psi)$ satisfy the differential equation

$$
\begin{aligned}
& \epsilon \gamma \dot{\Theta}=-g \cos (\Theta+\delta)-h \sin (\Theta+\delta) \\
& \epsilon \dot{\gamma}=-\left(1-\gamma^{2}\right)(g \sin (\Theta+\delta)-h \cos (\Theta+\delta)) \\
& \epsilon \gamma \dot{\Psi}=-\sqrt{1-\gamma^{2}}\left(g \cos (\Theta+\delta)+h(\sin (\Theta+\delta)), \quad \epsilon=K^{-1}\right.
\end{aligned}
$$

then the identities (13) reduce to

$$
\sqrt{1-\gamma^{2}} \dot{\psi}_{k}=\left(1-\gamma \cos \left(\psi_{k}-\Psi\right)\right) \omega_{k}, \quad k=1, \ldots, N
$$

These form $N$ trivial equations when $\omega_{k}=0$ for all $k$. For the system studied in [WS94] (with $\omega_{k} \equiv 0$ ), the change of coordinates (12) thus reveals that the original $N$ dimensional dynamics are in fact foliated by 3-dimensional invariant subspaces. When $|\epsilon|=1 /|K|$ is a small parameter, the system (14)(15) is singularly perturbed. In the new coordinates, the three fast dynamic variables are $(\Theta, \gamma, \Psi)$ and the $N$ slow variables are $\psi_{k}, 1 \leq k \leq N$. The fast dynamics are described by (14) with the slow variables frozen to their initial condition: $\psi_{k}(t)=\psi_{k}(0)$ for all $t \geq 0,1 \leq k \leq N$.

The analysis of the 3 -dimensional system (14) further reduces to a 2 dimensional analysis by observing that the variable $\Theta$ can be eliminated from the right-hand side of (14). Indeed one has

$$
\begin{aligned}
& \epsilon \dot{\gamma}=\left(1-\gamma^{2}\right) \operatorname{Im}\left(p_{\theta} e^{-i \Theta} e^{-i \delta}\right) \\
& \epsilon \gamma \dot{\Psi}=-\sqrt{1-\gamma^{2}} \operatorname{Re}\left(p_{\theta} e^{-i \Theta} e^{-i \delta}\right)
\end{aligned}
$$


but $p_{\theta} e^{-i \Theta}=\frac{1}{N} \sum e^{i\left(\theta_{k}-\Theta\right)}$ and $\theta_{k}-\Theta=2 \arctan \left(\sqrt{\frac{1+\gamma}{1-\gamma}} \tan \frac{\psi_{k}-\Psi}{2}\right)$. The fast system (14) thus decouples into the two-dimensional system (16) and an additional equation for the dynamics of $\Theta$ (which is in fact irrelevant for the convergence analysis).

Observe that when $p_{\theta}=0$ (i.e., on the balanced manifold), the dynamics (16) are at rest. Thus, under the assumption $\omega_{k} \equiv 0$ for all $k$, the balanced manifold is invariant.

To complete the (global) analysis of (16), Watanabe and Strogatz introduce the Lyapunov function

$$
V(\gamma, \Psi, \psi)=\frac{1}{N} \sum_{k=1}^{N} \log \left(\frac{1-\gamma \cos \left(\psi_{k}-\Psi\right)}{\sqrt{1-\gamma^{2}}}\right)
$$

which takes its minimium at $\gamma=0$, and show (when $\dot{\psi}_{k}=0$ ) that its timederivative satisfies

$$
\dot{V}=K\left|p_{\theta}\right|^{2} \sin \delta
$$

The behavior of the fast dynamics of (14)-(15) is thus entirely determined by the evolution of the scalar function $V$ in the invariant domain defined by $0 \leq \gamma<1$. The Lyapunov function $V$ is monotonically increasing along solutions when $K>0$ and monotonically decreasing along solutions when $K<0$. It is stationary only when the linear momentum $p_{\theta}$ vanishes.

In order to fully interpret this result, we first describe in Section 3.2 an additional constraint defined by Watanabe and Strogatz that makes the balanced manifold equivalent to the manifold $\gamma=0$. Under these conditions, it then follows that $\gamma=0$ is a stationary point of (16) (and this is equivalent to the invariance of the balanced manifold).

\subsection{Zero centroid constraint and interpretation of the dynamics}

Since the $N$-dimensional dynamics (8) are immersed in the $(N+3)$-dimensional dynamics (14)-(15), there are three degrees of freedom in choosing the initial condition $\psi_{k}(0)$ such as to satisfy (12). Further insight on the behavior of the solutions is gained by imposing the constraint

$$
p_{\psi}=\sum_{k=1}^{N} e^{i \psi_{k}}=0
$$

which fixes to zero the centroid of the new (phase) variables $\psi_{k}$. The change of coordinates (12) with (14) and the constraint (18) has the convenient interpretation of redistributing the phase variables $\theta_{k}$ on the unit circle in such a way that they become constants of motion (in the fast time scale or when $\omega_{k} \equiv 0$ ) with a vanishing centroid. (To make the change of coordinates oneto-one, a third algebraic constraint can be imposed, e.g. by fixing the initial 
condition $\Theta$ to an arbitrary value; again this is irrelevant to interpreting the behavior of the solutions).

The constraint (18) makes the balanced manifold equally determined by the condition $p_{\theta}=0$ or by the new condition $\gamma=0$. This is because, when $\gamma=0$, the change of coordinates becomes the linear transformation $(\bmod \pi)$

$$
\psi_{k}=\theta_{k}+\Psi-\Theta
$$

so that the centroid $p_{\psi}=\sum_{k} e^{i \psi_{k}}$ coincides with the centroid $p_{\theta}=\sum_{k} e^{i \theta_{k}}$. In the new coordinates (12) with (14) and (18), stabilizing the balanced manifold therefore becomes synonymous with stabilizing the manifold $\gamma=0$.

It is further shown in [WS94] that the Lyapunov function $V$, which vanishes at $\gamma=0$, monotonically increases to $+\infty$ as $\gamma \rightarrow 1$. Convergence of $\gamma(t)$ to 1 implies phase synchronization $\theta_{i}(t) \rightarrow \Theta(t)+\pi, 1 \leq i \leq N$, as shown by the identities

$$
\begin{aligned}
\sin \left(\theta_{k}-\Theta\right) & =\frac{\sqrt{1-\gamma^{2}} \sin \left(\psi_{k}-\Psi\right)}{1-\gamma \cos \left(\psi_{k}-\Psi\right)} \\
\cos \left(\theta_{k}-\Theta\right) & =\frac{\cos \left(\psi_{k}-\Psi\right)-\gamma}{1-\gamma \cos \left(\psi_{k}-\Psi\right)} .
\end{aligned}
$$

One concludes that the variable $0 \leq \gamma<1$ measures the synchronization of the phase variables: it is minimal on the balanced manifold and maximal when all the phases synchronize. The balanced manifold is globally attracting when $K<0$ and the synchronized state is globally attracting when $K>0$.

\subsection{Singularities}

Two types of singularities must be dealt with to complete the analysis of the model (8): the singularity of (14) at $\gamma=0$ and the singularities of the change of variables (12) under the constraint (18). The (apparent) singularity at $\gamma=0$ is due to the polar nature of the coordinates $(\gamma, \Theta)$ and is easily removed with an additional change of coordinates [WS94]. In contrast, a true singularity occurs in the change of coordinates (12), (18) for "majority clusters", i.e. initial conditions for which more than $N / 2$ of the phase variables are identical: in this case, more than $N / 2$ phase variables $\psi_{k}$ are also identical and the centroid $p_{\psi}$ cannot vanish. A special treatment is required for those special initial conditions but we refer the interested reader to [WS94].

\section{Linear momentum stabilization}

We now return to the singularly perturbed particle model

$$
\begin{aligned}
\dot{r}_{k} & =e^{i \theta_{k}} \\
\epsilon \dot{\theta}_{k} & =\epsilon u_{k}^{s p a c}+\frac{1}{N} \sum_{j=1}^{N} \sin \left(\theta_{j}-\theta_{k}\right), \quad 1 \leq k \leq N
\end{aligned}
$$

and interpret the conclusions of the analysis in the previous section to its fast subsystem 


$$
(\operatorname{sign} \mathrm{K}) \dot{\theta}_{k}=\frac{1}{N} \sum_{j=1}^{N} \sin \left(\theta_{j}-\theta_{k}\right), \quad 1 \leq k \leq N .
$$

If $K>0$, all the solutions of (21) asymptotically synchronize. For the singularly perturbed model (20), the conclusion is that for $K$ large enough, all solutions converge in the fast time scale $\frac{t}{\epsilon}$ to an invariant slow manifold where the motion is nearly parallel. The slow manifold of parallel motion has codimension $N-1$; in the asymptotic limit $\epsilon=0$, it is determined by the $N-1$ algebraic conditions

$$
\theta_{k}=\theta_{1}, \quad 1<k \leq N
$$

In contrast, if $K<0$, then the solutions of (21) converge to the balanced manifold characterized by $p_{\theta}=0$. For the singularly perturbed model (20), the conclusion is that for $|K|$ large enough, all solutions converge in the fast time scale $\frac{t}{\epsilon}$ to an invariant slow manifold where the center of mass is nearly fixed. This slow manifold has codimension 2; in the asymptotic limit $\epsilon=0$, it is determined by the two algebraic conditions

$$
\sum_{k=1}^{N} \cos \theta_{k}=\sum_{k=1}^{N} \sin \theta_{k}=0 .
$$

In the next two sections, we illustrate how the slow dynamics can be designed to stabilize relative equilibria of (20).

\section{Stabilization of parallel formations}

To analyze the slow dynamics of the singularly perturbed particle model (5) in the case $\epsilon=1 / K>0$, we determine the first-order approximation of its slow manifold. The slow manifold has the expression

$$
\theta_{k}=\theta_{1}+h_{k}(r, \epsilon), \quad 1<k<N
$$

To determine the functions $h_{k}, 1<k<N$, one expresses the invariance of the manifold

$$
\theta_{k}-\theta_{1}=h_{k} \Rightarrow \dot{\theta}_{k}-\dot{\theta}_{1}=\dot{h}_{k}
$$

which determines the functions $h_{k}$ as the solutions of the PDE

$$
\begin{gathered}
\left(\epsilon\left(\dot{\theta}_{k}-\dot{\theta}_{1}\right)=\right) \epsilon\left(u_{k}^{\text {spac }}-u_{1}^{s p a c}\right)+\left|p_{\theta}\right|\left(\sin \left(\phi-\theta_{1}-h_{k}\right)-\sin \left(\phi-\theta_{1}\right)\right) \\
=\epsilon \sum_{j} \frac{\partial h_{k}}{\partial r_{j}} e^{i\left(\theta_{1}+h_{j}\right)}\left(=\epsilon \dot{h}_{k}\right)
\end{gathered}
$$

Expanding $h_{k}(r, \epsilon)=\epsilon h_{k}^{1}(r)+O\left(\epsilon^{2}\right)$ and equating the first-order terms in $\epsilon$ in (22) yields the first-order approximation

$$
h_{k}(r, \epsilon)=\epsilon\left(u_{k}^{s p a c}-u_{1}^{s p a c}\right)+O\left(\epsilon^{2}\right), \quad 1<k \leq N .
$$


The slow dynamics are obtained by substituting the approximation (23) in (5):

$$
\dot{r}_{k}=e^{i \theta_{1}}\left(1+i \epsilon\left(u_{k}^{s p a c}-u_{1}^{s p a c}\right)\right)+O\left(\epsilon^{2}\right) .
$$

For the difference $r_{k j}=r_{k}-r_{j}$, this yields the slow dynamics

$$
\dot{r}_{k j}=\epsilon i e^{i \theta_{1}}\left(u_{k}^{s p a c}-u_{j}^{s p a c}\right)+0\left(\epsilon^{2}\right) .
$$

The controls $u_{k}^{\text {spac }}, 1<k \leq N$ can now be determined to assign formations for the group of particles moving in parallel. As an illustration, we follow the approach proposed in [BL02] to stabilize formations of (non-oriented) particles: the desired formation is specified by the critical points of a scalar potential

$$
U=\sum_{i=1}^{N} \sum_{j>i} U_{I}\left(r_{i j}\right)
$$

where the potential $U_{I}\left(r_{i j}\right)=U_{I}\left(r_{i}-r_{j}\right)=U_{I}\left(r_{j i}\right)$ determines the desired interaction from particle $j$ on particle $i$. For instance, the choice

$$
U_{I}\left(r_{i j}\right)=\log \left\|r_{i j}\right\|+\frac{d_{0}}{\left\|r_{i j}\right\|}
$$

results in the feedback

$$
\nabla U_{I}=\frac{\partial U_{I}}{\partial r_{i}}=\left(\frac{1}{\left\|r_{i j}\right\|}-\frac{d_{0}}{\left\|r_{i j}\right\|^{2}}\right) \frac{r_{i j}}{\left\|r_{i j}\right\|}
$$

from particle $j$ on particle $i$ which vanishes only at the equilibrium distance $\left\|r_{i j}\right\|=\left\|r_{i}-r_{j}\right\|=d_{0}$. Gradient-like dynamics for the slow system (24) are obtained with the feedback control

$$
u_{k}^{s p a c}=-\sum_{j \neq k}<\nabla U_{I}\left(r_{k j}\right), i e^{i \theta_{k}}>
$$

which causes the scalar potential $U$ to decrease along the solutions in the slow manifold:

$$
\begin{aligned}
\dot{U} & =\sum_{k=1}^{N} \sum_{j>k} \nabla U_{I}\left(r_{k j}\right) \dot{r}_{k j} \\
& =\epsilon \sum_{k=1}^{N} \sum_{j>k}<\nabla U_{I}\left(r_{k j}\right), i e^{i \theta_{1}}>\left(u_{k}^{s p a c}-u_{j}^{s p a c}\right)+O\left(\epsilon^{2}\right) \\
& =-\epsilon \sum_{k=1}^{N}\left(u_{k}^{s p a c}\right)^{2}+O\left(\epsilon^{2}\right) .
\end{aligned}
$$

Equilibrium configurations that minimize the potential $U$ favor uniform spacing between the particles. See Figure 1. 
(1.a)
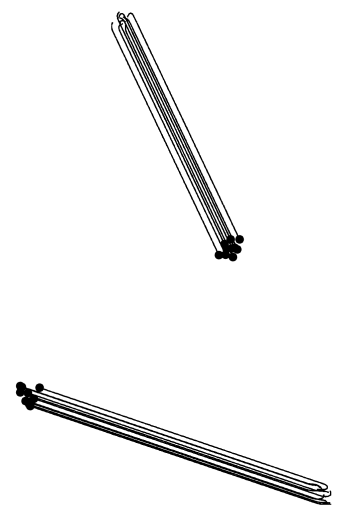

(2.a) (1.b)
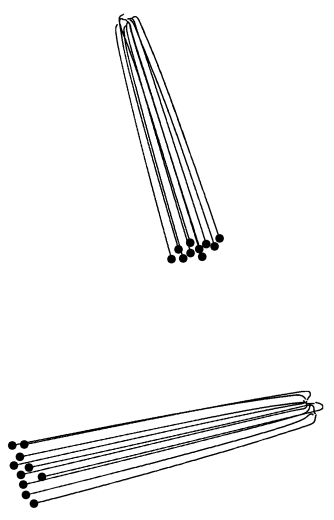

Fig. 1. Parallel motion for ten vehicles for two sets of random initial conditions (1) and (2). No spacing control is used in (1.a) and (2.a). In (1.b) and (2.b), $u_{k}^{\text {spac }}$ is given by (28).

\section{Controlled-invariance of the balanced manifold and stabilization of circular motion}

To analyze the slow dynamics of the singularly perturbed particle model (5) in the case $\epsilon=1 / K<0$, we use the new coordinates introduced in Section 3:

$$
\begin{aligned}
\dot{r}_{k} & =e^{i \theta_{k}} \\
\sqrt{1-\gamma^{2}} \dot{\psi}_{k} & =\left(1-\gamma \cos \left(\psi_{k}-\Psi\right)\right) u_{k}^{s p a c}, \quad k=1, \ldots, N \\
\epsilon \gamma \dot{\Theta} & =-g \cos (\Theta+\delta)-h \sin (\Theta+\delta)) \\
\epsilon \dot{\gamma} & =-\left(1-\gamma^{2}\right)(g \sin (\Theta+\delta)-h \cos (\Theta+\delta)) \\
\epsilon \gamma \dot{\Psi} & =-\sqrt{1-\gamma^{2}}\left(g \cos (\Theta+\delta)+h(\sin (\Theta+\delta)), \quad \epsilon=K^{-1}<0\right.
\end{aligned}
$$

The conclusions of Section 3 for the fast subsystem of (29) rely on the constraint $p_{\psi}=0$ (constraint (18)). Under this constraint, the balanced manifold was shown to be equally determined by the condition $p_{\theta}=0$ (in the old coordinates) or by the condition $\gamma=0$ (in the new coordinates). Moreover, the balanced manifold was shown to be invariant for the solutions of (29) when $u_{k}^{s p a c}=0$.

All these properties can be retained with additional nontrivial control $u_{k}^{s p a c}$ provided we restrict $u_{k}^{s p a c}$ to satisfy the constraint

$$
\frac{1}{N} \sum_{k=1}^{N} e^{i \theta_{k}} u_{k}^{s p a c}=0
$$

Observe from (5) and (11) that $p_{\theta}=0$ implies that $\dot{\theta}_{k}=u_{k}^{s p a c}$. Thus, condition (30) enforces the (controlled) invariance of the balanced manifold: 


$$
p_{\theta}=0 \Rightarrow \dot{p}_{\theta}=\frac{i}{N} \sum_{k=1}^{N} e^{i \theta_{k}} \dot{\theta}_{k}=\frac{i}{N} \sum_{k=1}^{N} e^{i \theta_{k}} u_{k}^{s p a c}=0 \text {. }
$$

But the constraint (30) also projects the dynamics (29) onto the manifold $p_{\psi}=0$. In other words, it renders the dynamics (29) compatible with the change of coordinates (12)-(18). Indeed, one verifies that (29) together with the condition (30) imply the invariance of $p_{\psi}=0$ :

$$
\begin{aligned}
\sum_{k} e^{i \theta_{k}} u_{k}^{s p a c}=0 \Rightarrow \sum_{k} e^{i\left(\theta_{k}-\Theta\right)} u_{k}^{s p a c}=0 \\
\Rightarrow \sum_{k} \sin \left(\theta_{k}-\Theta\right) u_{k}^{s p a c}=\sum_{k} \cos \left(\theta_{k}-\Theta\right) u_{k}^{s p a c}=0 \\
\Rightarrow{ }^{(19)} \sum_{k} \sin \left(\psi_{k}-\Psi\right) \dot{\psi}_{k}=\sum_{k} \cos \left(\psi_{k}-\Psi\right) \dot{\psi}_{k}=0 \\
\Rightarrow \sum_{k} e^{i \psi_{k}} \dot{\psi}_{k}=\dot{p}_{\psi}=0 .
\end{aligned}
$$

Under the constraint (30), the balanced manifold thus becomes the (exact) slow manifold of the singularly perturbed system (29). The dynamics in the slow manifold reduce to

$$
\begin{aligned}
& \dot{r}_{k}=e^{i \theta_{k}}=e^{i\left(\psi_{k}+\Theta-\Psi\right)} \\
& \dot{\psi}_{k}=u_{k}^{s p a c}, \quad k=1, \ldots, N \\
& \sum_{k=1}^{N} e^{i \theta_{k}}=\sum_{k=1}^{N} e^{i \psi_{k}}=0 .
\end{aligned}
$$

It can be seen that the design of the particle model on the slow manifold is the design of the original particle model assuming a center of mass at rest.

We will illustrate below how being able to make this assumption simplifies the design of collective motions around a fixed point. The design of the complete particle model is thus decomposed in two phases: (1) the design of a reduced control $u^{\text {bal }}$ under the assumption of a fixed center of mass, that is, restricted to the balanced manifold, and (2) the design of a complete control $u$ which reduces to $u^{b a l}$ in the balanced manifold and at the same time enforces convergence to the balanced manifold. For $N \geq 3$, the full control is deduced from the reduced control by the expression

$$
u=u^{s p a c}+K u^{a l i g n}=\left(I-P_{\theta}\left(P_{\theta}^{T} P_{\theta}\right)^{-1} P_{\theta}^{T}\right) u^{b a l}+K u^{a l i g n}, \quad K<<0
$$

where $P_{\theta}$ denotes the $N \times 2$ matrix with first column $\frac{\cos \theta}{N}$ and second column $\frac{\sin \theta}{N}$. The projector $I-P_{\theta}\left(P_{\theta}^{T} P_{\theta}\right)^{-1} P_{\theta}^{T}$ enforces the constraint (30) which, in matrix notation, reads $P_{\theta}^{T} u^{s p a c}=0$.

\subsection{Stabilization of a circular relative equilibrium}

As an illustration of the above design procedure, we consider the stabilization of the group of particles on a circle of fixed radius $\rho_{0}$ centered at the (fixed) center of mass. 
The design of a circular motion for a single particle with coordinates $\left(r_{k}, \theta_{k}\right)$ around a fixed beacon $R$ has been addressed in [JK02]. The authors propose (a variant of) the feedback control

$$
u_{k}^{b a l}=-f\left(\rho_{k}\right)<\frac{\tilde{r}_{k}}{\rho_{k}}, i e^{i \theta_{k}}>-<\frac{\tilde{r}_{k}}{\rho_{k}}, e^{i \theta_{k}}>
$$

with $\tilde{r}_{k}=r_{k}-R$ and $\rho_{k}=\left\|\tilde{r}_{k}\right\|$. See Figure 2 for an illustration. The

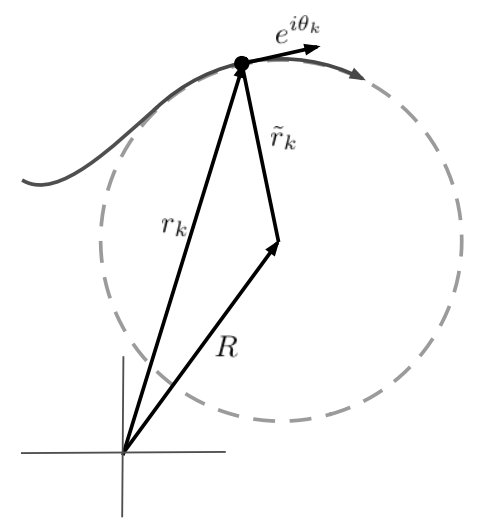

Fig. 2. Circular motion of a single particle around the fixed beacon $R$

second term of the control law (33) stabilizes circular motions: it vanishes when the velocity vector is orthogonal to the relative position vector. The function $f(\cdot)$ in (33) plays the same role as (27) in the parallel control of the previous section, creating an attractive interaction when the distance $\rho_{k}$ exceeds the equilibrium distance $d_{0}$ and a repulsive interaction otherwise. (The choice $f\left(\rho_{k}\right)=1-\left(d_{0} / \rho_{k}\right)^{2}$ is proposed in [JK03]). With the control (33), the Lyapunov function

$$
U_{k}=-\log \left|<\frac{\tilde{r}_{k}}{\rho_{k}}, i e^{i \theta_{k}}>\right|+\int_{\bar{\rho}}^{\rho_{k}}\left(f(s)-\frac{1}{s}\right) d s
$$

has a global minimum at a relative equilibrium which corresponds to circular motion around the fixed beacon: the equilibrium is determined by a velocity vector orthogonal to the relative position vector (i.e. $\left\langle\tilde{r}_{k}, e^{i \theta_{k}}\right\rangle=0$ ), and a distance $\bar{\rho}$ to the beacon such that $f(\bar{\rho})=\frac{1}{\bar{\rho}}$. Assuming $\dot{R}=0$, the timederivative of $U_{k}$ satisfies

$$
\dot{U}_{k}=-<\frac{\tilde{r}_{k}}{\rho_{k}}, e^{i \theta_{k}}>^{2} /<\frac{\tilde{r}_{k}}{\rho_{k}}, i e^{i \theta_{k}}>
$$

The Lyapunov function $U_{k}$ provides a global convergence analysis for the single particle model. In particular, it can be used to prove asymptotic stability of the clokwise rotation around the fixed beacon. 
The design of circular motion for $N$ particles in the balanced manifold is an immediate extension of the single particle design: we apply (33) to each particle $1 \leq k \leq N$, with the fixed beacon replaced by the center of mass of the group $R=\frac{1}{N} \sum_{k} r_{k}$. Under the constraint $\dot{R}=p_{\theta}=0$, the Lyapunov function $U=\sum_{k} U_{k}$ provides the same global convergence analysis for the group of particles as for a single particle.

Inserting the control law (33) in the general formula (32) thus yields a stabilizing control law for the original particle model (1). The conclusions of the asymptotic analysis, which assumes large values for the parameter $|K|$, seem well retained in simulations even when a time-scale separation is not enforced between the fast stabilization of the center of mass and the slow stabilization of the circular formation in the balanced manifold. See Figure 3.
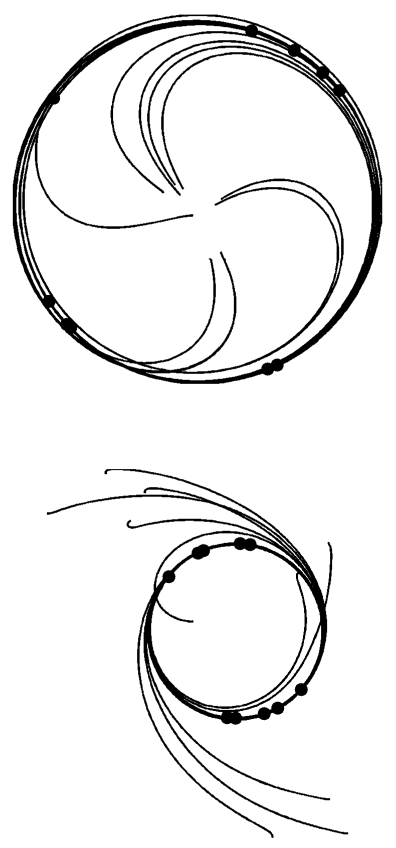

Fig. 3. Circular motion with $K=-1$ for two sets of random initial conditions.

We finish this section with a remark on the controlled invariance of the balanced manifold. Exact invariance of the balanced manifold is convenient for the Lyapunov analysis of the closed-loop system and for the design of the slow dynamics but not required for the convergence of solutions to the desired circular motion. Not surprisingly, simulations of the particle model with the 
control law (32)-(33) without the projector $\left(I-P_{\theta}\left(P_{\theta}^{T} P_{\theta}\right)^{-1} P_{\theta}^{T}\right)$ suggest nearinvariance of the balanced manifold. Near-invariance of the balanced manifold in the absence of the constraint (30) is illustrated in Figure 4.

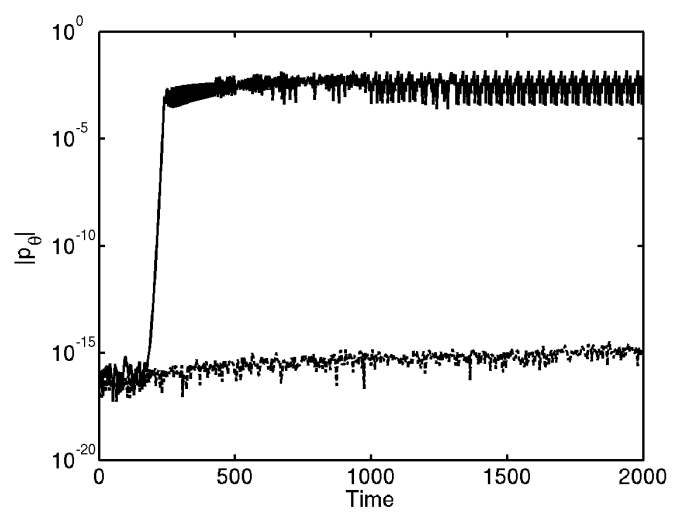

Fig. 4. Time evolution of the linear momentum $\left|p_{\theta}\right|$ for the control law (32)-(33), with (dashed) and without (plain) the projector $I-P_{\theta}\left(P_{\theta}^{T} P_{\theta}\right)^{-1} P_{\theta}^{T}(N=20$ and $\left.\left|p_{\theta}\right|(0)=0\right)$.

\section{Conclusions and directions for future work}

The present paper provides a global convergence analysis for the stabilization of relative equilibria in a simple particle model of self-propelled particles. The proposed approach rests on a two-time scale decomposition of the dynamics that decouples the stabilization of orientation variables from the stabilization of spacing variables. The fast dynamics analysis exploits previous results of the literature for models of phase oscillators. All-to-all sinusoidal coupling of the relative orientations results in stabilization of the linear momentum either to a maximal value (resulting in parallel motion) or to a minimal value (resulting in motion around a fixed center of mass). The slow dynamics analysis also exploits previous results in the literature to achieve parallel motion with prescribed formations or motion of all the particles on a fixed circle.

Natural extensions of the results would include analogous results for discrete-time models such as (7) or for the continuum limit of an infinite number of particles (Results for the continuum limit are included in the analysis of Watanabe and Strogatz[WS94]).

An important limitation of the present paper is the assumption of all-to-all coupling. This assumption is unrealistic in large groups of organisms and requires a prohibitively demanding communication topology in engineering applications. The results presented are likely to hold under relaxed assumptions 
on the network connectivity but extending the analysis to these situations is not straightforward. Recent convergence results for the Viczek model under weak connectivity assumptions [JLM02] provide new analysis tools for such generalizations.

The stabilization problems treated in this paper are considered a preliminary step to more challenging design problems such as path planning or collective optimization tasks. Both the Lie group structure of the model (1) and the time-scale decomposition of the overall dynamics into the control of aggregated parameters (such as the group linear momentum) and the control of individuals relative to these parameters might prove useful in such extensions.

\section{References}

[BL02] R. Bachmayer and N. E. Leonard, Vehicle networks for gradient descent in a sampled environment, Proc. of the 41st IEEE Conference on Decision and Control (2002).

$\left[\mathrm{CKJ}^{+}\right.$02] I.D. Couzin, J. Krause, R. James, G. Ruxton, and N. Franks, Collective memory and spatial sorting in animal groups, J. Theor. Biology (2002), no. $218,1-11$.

[EK90] B. Ermentrout and N. Kopell, Oscillator death in systems of coupled neural oscillators, SIAM J. Appl. Math.. 50 (1990), 125-146.

[HI97] F. Hoppensteadt and E. Izhikevich, Weakly connected neural networks, Springer-Verlag, New-York, 1997.

[II03] Autonomous Ocean Sampling Network II, http://www.princeton.edu/ dcsl/aosn/, 2003.

[JK02] E. Justh and P. Krishnaprasad, A simple control law for UAV formation flying, Technical Report 2002-38, http://www.isr.umd.edu.

[JK03] Steering laws and continum models for planar formations, To appear in Proc. of the 42nd IEEE Conference on Decision and Control (2003).

[JLM02] A. Jadbabaie, J. Lin, and A. S. Morse, Coordination of groups of mobile autonomous agents using nearest neighbor rules, IEEE Trans. Automatic Control 48 (2002), 988-1001.

[Kur84] Y. Kuramoto, Chemical oscillations, waves, and turbulence, SpringerVerlag, Berlin, 1984.

[LRC01] H. Levine, W.-J. Rappel, and I. Cohen, Self-organization in systems of self-propelled particles, Physical Review E 63 (2001), 017101.

[Str00] S. Strogatz, From Kuramoto to Crawford: Exploring the onset of synchronization in populations of coupled oscillators, Physica D 143 (2000), 1-20.

$\left[\right.$ VCBJ $^{+}$95] T. Viczek, A. Czirok, E. Ben-Jacob, I. Cohen, and O. Shochet, Novel type of phase transition in a system of self-driven particles, Physical Review Letters 75 (1995), no. 6, 1226-1229.

[WS94] S. Watanabe and S. Strogatz, Constants of the motion of superconducting Josephson arrays, Physica D 74 (1994), 195-253. 